\title{
MassiveSumm: a very large-scale, very multilingual, newswire summarisation dataset
}

\author{
Daniel Varab and Natalie Schluter \\ IT University of Copenhagen \\ Denmark \\ \{djam, natschluter\}@itu.dk
}

\begin{abstract}
Current research in automatic summarisation is unapologetically anglo-centered-a persistent state-of-affairs, which also predates neural net approaches. High-quality automatic summarisation datasets are notoriously expensive to create, posing a challenge for any language. However, with digitalisation, archiving, and social media advertising of newswire articles, recent work has shown how, with careful methodology application, large-scale datasets can now be simply gathered instead of written. In this paper, we present a large-scale multilingual summarisation dataset containing articles in 92 languages, spread across 28.8 million articles, in more than 35 writing scripts. This is both the largest, most inclusive, existing automatic summarisation dataset, as well as one of the largest, most inclusive, ever published datasets for any NLP task. We present the first investigation on the efficacy of resource building from news platforms in the low-resource language setting. Finally, we provide some first insight on how low-resource language settings impact state-of-the-art automatic summarisation system performance.
\end{abstract}

\section{Introduction}

Automatic summarisation datasets are generally expensive to create, because they generally involve a human reading a document several times and then crafting a fluent piece of text that captures both the important information of the document and the intention of the resulting summary. Each datapoint in such a dataset could take hours to manually create. With digitalisation, archiving, and social media advertising of newswire articles, recent work has shown how, with dedicated time and methodology application, large-scale datasets can now be simply gathered instead of written (Grusky et al., 2018; Hermann et al., 2015). But the method development was carried out over English, and until the research presented here, the method has only been applied to a very limited number of relatively richly-resourced languages (Varab and Schluter, 2020; Scialom et al., 2020).

We have extended the methodology further (Section 3) and applied it carefully and widely to generate MassiveSumm: a very large-scale, very multilingual summarisation dataset of 28.8 million articles, containing data in 92 languages, using more than 35 writing scripts. This is by far both the largest, most inclusive, existing automatic summarisation dataset, as well as one of the largest, most inclusive, ever published datasets for any NLP task. The bulk of this paper outlines the size, diversity and inclusivity of the dataset as an automatic summarisation dataset, as well as simply raw text data in comparison with two other multilingual large-scale widely used datasets in NLP: Wikipedia and Common Crawl (Section 4).

In light of extending and applying the data acquisition method under the low-resource setting, we identify some unreasonable conditions for language inclusion in automatic summarisation research, which stand to perpetuate a lack of language diversity in system development and therefore unequal access to these tools. We also present some experimental evidence that failure to include a more diverse set of language data in automatic summarisation research can result in only very language specific system design when language agnostic design has been claimed (Section 5).

\section{Related Work}

A number of works presenting large-scale datasets for automatic summarisation have been presented in the past couple of years. We survey this work here to provide some research context for MassiveSumm.

The New York Times Corpus (NYT) consists of 1.8 million articles from the New York Times (Sandhaus, 2008) between 1987 and 2007. The automatic summarisation portion of this dataset 
consists of 650,000 article-summary pairs, where the summaries are written by library scientists. Unlike the rest of the datasets discussed in this section, NYT is created and maintained by the platform that the articles belong to.

The CNN/Daily Mail (CNNDM) dataset (Hermann et al., 2015) is an English language automatically acquired Question Answering dataset composed of newswire articles and their corresponding highlights from two separate platforms: cnn. com and dailymail.co.uk. The dataset was later converted into a summarisation dataset by concatenating these article highlights into article summaries (Cheng and Lapata, 2016; Nallapati et al., 2016). The summarisation dataset consists of 312,000 summary-article pairs. It has become the most broadly used automatically collected English summarisation dataset.

With the same methodology as CNNDM, Narayan et al. (2018) collected the XSum dataset of approximately 230,000 summary-article pairs from the bbc . com news platform. And Scialom et al. (2020) collected the MLSum dataset for five languages from five corresponding news platforms: French, German, Spanish, Russian, Turkish, catering their platform dependent method to each separate news platform. The resulting datset contains a total of around 1.5 million article-summary language pairs. MLSum was the first large-scale multilingual dataset, but all five of the languages of the dataset were still European, Indo-European, and relatively high-resourced within NLP. We note that while, similarly to XSum, MassiveSumm also contains article-summary pairs from the bbc. com platform, there are two important differences which make for zero overlap between the two datasets: (1) we include no English datapoints in our dataset, and (2) our summaries are not article highlights, but social media article descriptions, as is done for the remaining newswire datasets surveyed here.

The Newsroom dataset (Grusky et al., 2018) is the first large-scale English dataset generated specifically for automatic summarisation. The key insight into automatically creating this dataset was in observing use of a social media standard, called Open $\mathrm{Graph}^{1}$, by publishers to improve their search engine results. According to this standard, a description of the article contents, used for advertising on social media, should be recorded in the mark-up of the article's web page. The method

\footnotetext{
${ }^{1}$ https: / / ogp.me/
}

allowed for scraping news articles from any news outlet, so long as the news outlet upheld the social media standard. Hence, by contrast to the method for acquiring the CNNDM, Newsroom's method was website agnostic, which meant that scraping was no longer constrained to collecting data from specific platforms. Grusky et al. (2018) created Newsroom by conducting a scrape of news articles from 38 English language news outlets spanning two decades starting from the late 1990s, when news platforms first began digitalising their content widely, to 2017. The dataset contains 1.3 million document summary pairs.

Varab and Schluter (2020) extend, streamline and improve the Newsroom methodology to assemble the first automatic summarisation dataset for Danish, DaNewsroom. Their work comprises the first non-English website agnostic approach to large-scale article-summary collection, across 19 Danish news platforms and resulting in a dataset of $1.1 \mathrm{M}$ article-summary pairs. The methodology of this paper is adapted from this extension of the Newsroom methodology.

Related to this, the GlobalVoices dataset (Nguyen and Daumé III, 2019), is an automatic summarisation dataset across 15 languages from one single platform, https:// globalvoices.org. Although its original collection is similar to Newsroom and DaNewsroom, the resulting dataset is relatively small with less than 30,000 article-summary pairs across all languages in total, including English. Moreover, approximately 800 English summaries are further crowdsourced. The dataset contains purely parallel data and its intended use is for cross-lingual summarisation. MassiveSumm most likely includes all non-English datapoints scraped for GlobalVoices, as this was one of the hundreds of its news platform data sources.

Two further large-scale datasets are not based on newswire. (1) BigPatent (Sharma et al., 2019) consists of 1.3 million U.S. patent English language abstract-document pairs, written between 1971 and 2018, across nine technological areas, all from the Google Patents Public Datasets (Google, 2018). (2) LCSTS The Large Scale Chinese Short Text Summarization Dataset (Hu et al., 2015) consists of 2.4 million text-summary pairs from the Sina Weibo microblogging platform, where post texts are paired with summaries provided by the author of each text. 
Contemporaneously to our work, Hasan et al (2021) developed XL-Sum, a summarisation dataset from the BBC news platform. However, their work covers less than a twelfth of the articlesummary pairs: around 1 million across 44 languages and a single news platform, compared with our 12.3 million across 92 languages and 370 news platforms.

\section{Methodology}

Our methodology consists of roughly three parts: (1) manual annotation, (2) automatic collection, and (3) quality control. The first part is unique to the dataset presented here and represents a workintensive annotation process which seeks to ensure both breadth in terms of language inclusivity, quality and consistency of the data. The remaining parts are measured adjustments of the prior extensions of Grusky et al. (2018)'s methodology by Varab and Schluter (2020).

Manual annotation. We first compiled a list of languages to be represented in the dataset. Our goal was to cover as many languages as possible, with a prioritisation of breadth, linguistic diversity, and language inclusivity, over depth. Then we manually searched for as many news platforms as possible for each language, by contrast to Grusky et al. (2018) who collected news platforms from publicly available lists.

For each news platform we required either (1) that it published exclusively in the language we had associated with it, or (2) published in way such that we could reliably distinguish the difference between languages later on (for example, the platform identified the languages for us). All other platforms were discarded.

Having determined which news platform were suitable language-wise, the next step was to manually investigate which platforms were technically suitable: we required these platforms to point to explicit lists of articles on their platform to avoid non-article content such as frontpages, albums or videos. In total, 370 different platforms met our requirements and were retained.

Automatic collection. With the list of suitable news platforms, we obtained all article URLs for each platform by retrieving them from archive. org. This is a slow process.

Having had collected the URLs for each platform we observe a significant difference between the amount of URLs across languages, some in the tens of millions, some in the thousands. We stored article URLs of the language together in language bins. We shuffled each bin and proceed to sample an equal amount of URLs from each bin and output them to a download queue. This allowed us to ensure that less frequent languages would always be scraped at the same priority as more frequent ones. Less frequent languages were sampled until they were exhausted, and thus over represented languages were sub-sampled.

Quality Control. We carry out a number of automatic checks for quality control, similarly to Varab and Schluter (2020). The number of articles filtered out of the dataset due to these checks can be seen in Table 1. In particular, we filter out articles with no contents, summaries with no contents, summaries that are prefixes of the article body, and summaries that are prefixes followed by “...”. We quantify this filtering process in Section 4.

Distribution. Practically speaking, the publically available dataset is distributed as a list of urls for each language (split into train/dev/test sets) and a single software package for downloading and processing the web pages. ${ }^{2}$

\section{The numbers}

Total counts. We refer to Table 1. Over 31 million articles were scraped from 370 news platforms, across 92 languages, from 38 language genera withing the following 16 language families: (1) Indo-European, (2) Afro-Asiatic, (3) Mande, (4) Niger-Congo, (5) Austronesian, (6) Altaic, (7) Sino-Tibetan, (8) Austro-Asiatic, (9) Kartvelian, (10) Uralic, (11) Japanese, (12) Dravidian, (13) Korean, (14) Tai-Kadai, (15) other, for Haïtian, and (16) Aymaran. ${ }^{3}$ Of these, approximately 3 million scraped article pages had an empty article $(2,981,925)$ and were filtered from the dataset, leaving over 28.8 million articles of raw multilingual text data, which we refer to as MassiveSumm-All.

As explained in Section 3, a number of filters were applied to the dataset to improve its quality for automatic summarisation. In particular, we did a check to ensure that summaries were neither empty nor just prefixes of the article, so that the

\footnotetext{
${ }^{2}$ https://github.com/danielvarab/ massive-summ

${ }^{3}$ We took language family definitions and genus definitions from https: //wals.info/ database.
} 

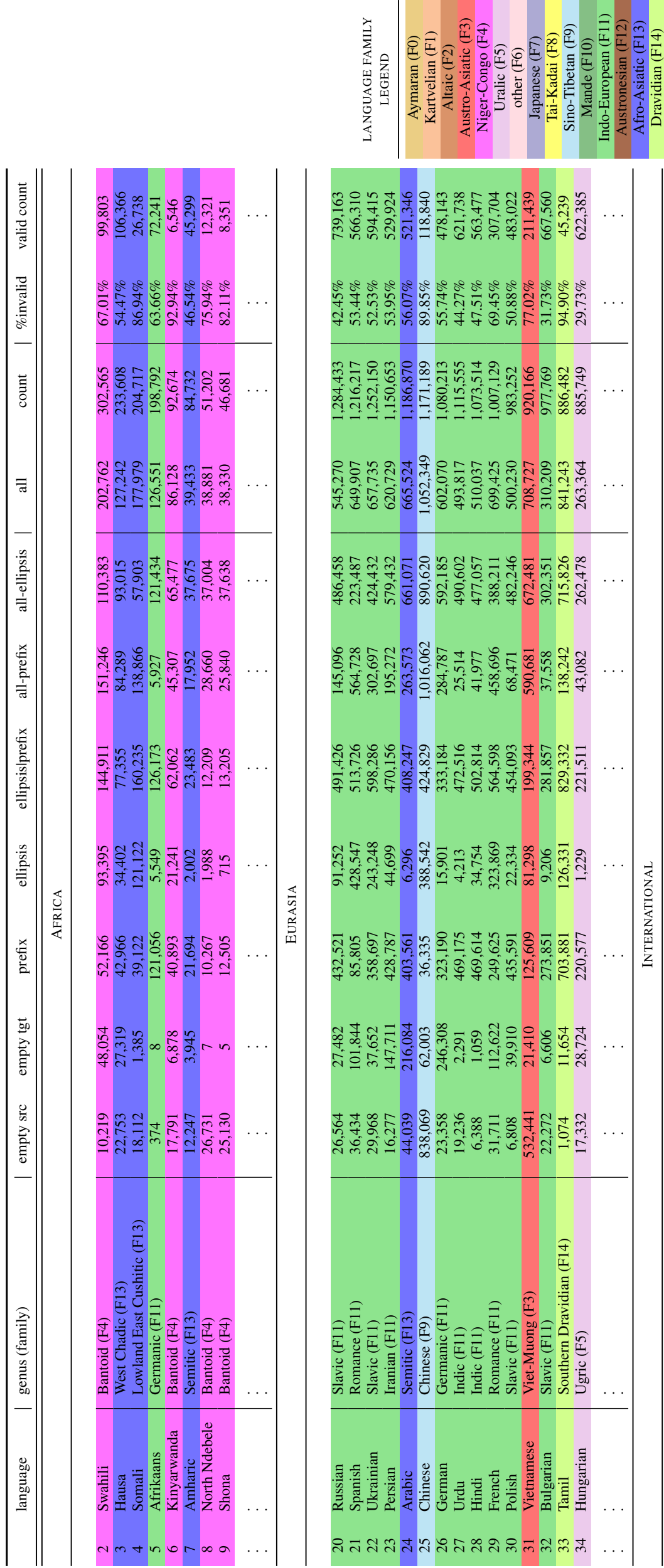

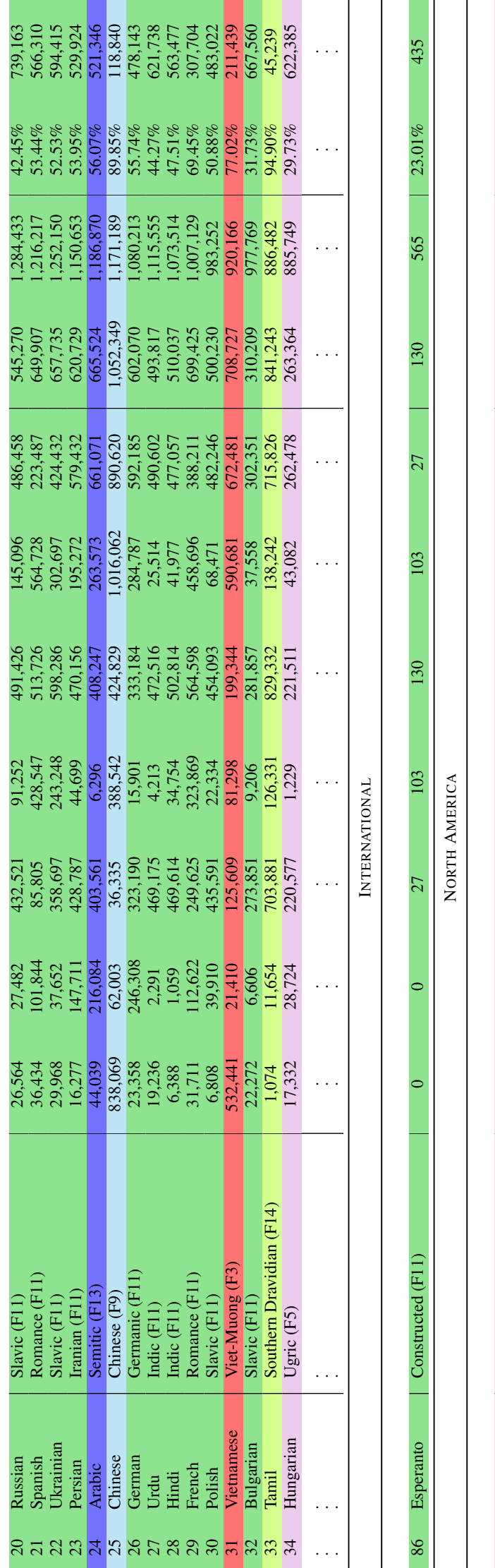

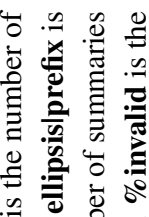

ध : हो

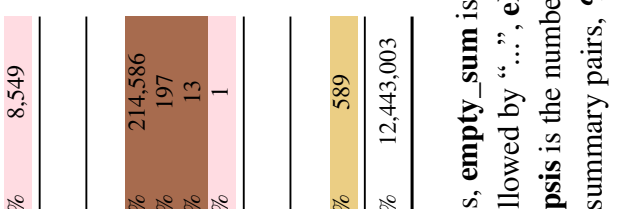

s 递产 至

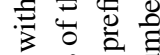

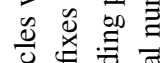
氖总吾 항 可 可

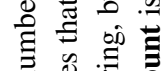

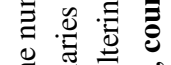
웜 足言离 ज.

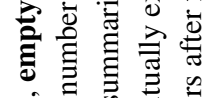

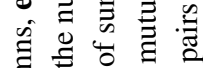
言

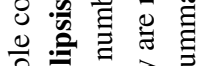

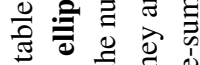
过

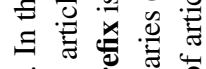

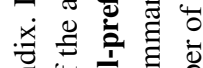

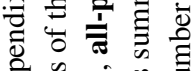

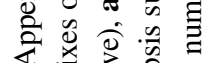

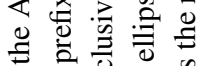
: 0 $\infty \frac{\pi}{\sigma}$ 흥 可

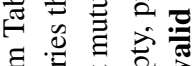

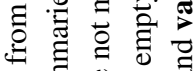

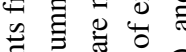
言 言它 至 ह 2.

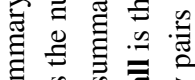

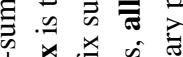

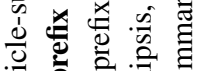
氖完言 跣

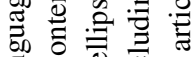

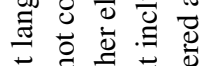

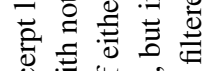

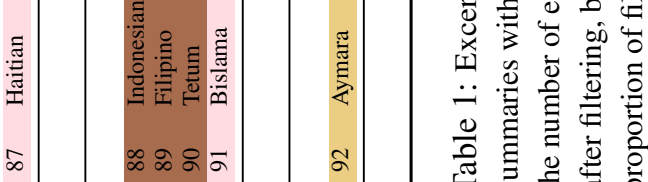


resulting dataset did not include trivial instances for system development. MassiveSumm can therefore be seen under two views: MassiveSumm-All (MS-All) which consists of all non-empty articles (and any available summaries) before application of the above-mentioned filters. And a subset of thisthe MassiveSumm (MS) summarisation dataset intended for automatic summarisation system development; this dataset is the result of the application of the filters.

We observe (Table 2) that the majority of the dataset, approximately 16.5 million articlesummary pairs, did not survive the summary quality control filtering process. The result was $12,368,113$ article-summary pairs surviving a minimal quality control for utility in automatic summarisation system development, of which the automaticsummarisation dataset portion of MassiveSumm consists.

\begin{tabular}{|c|c|c|}
\hline dataset & description & size \\
\hline $\begin{array}{l}\text { MassiveSumm } \\
\text { (MS) }\end{array}$ & $\begin{array}{l}\text { Fully filtered automatic } \\
\text { summarisation data. }\end{array}$ & $\begin{array}{l}12,368,113 \\
\text { article-summary } \\
\text { pairs. }\end{array}$ \\
\hline $\begin{array}{l}\text { MassiveSumm- } \\
\text { All (MS-All) }\end{array}$ & $\begin{array}{l}\text { All non-empty articles } \\
\text { scraped. }\end{array}$ & $\begin{array}{l}28,879,290 \text { arti- } \\
\text { cles. }\end{array}$ \\
\hline
\end{tabular}

Table 2: Summary of the contents of MassiveSumm.

This filtering process resulted in a handful of languages having virtually no presence in the automatic summarisation portion of MassiveSumm. For instance, over $98.7 \%$ of Xhosa article-summary pairs were filtered out of the summarisation portion of the dataset, leaving only 172 instances.

Table 3 gives an overview of the article/articlesummary pair counts. We note that the IndoEuropean languages provide the majority of the data in the dataset. The Uralic family (here, only with Hungarian) is also relatively heavily represented in the dataset. The 10 Niger-Congo languages as a whole have less data than a single IndoEuropean language on average. In Section 5 we discuss why our current methodology can only result in perpetuating such under-representation in dataset quantities.

Comparing with web-scrape multilingual datasets. We compared the intersection of our dataset with two large-scale web datasets widely used by the NLP community: Wikipedia ${ }^{4}$ and

\footnotetext{
${ }^{4}$ https://en.wikipedia.org/wiki/List_ of_Wikipedias\#Edition_detailsasofMay $1 \overline{0}$, 2021
}

Common $\mathrm{Crawl}^{5}$. An overview of this comparison can be found in Table 4. The manual care that we took in curating the list of platforms from which we wanted to collect data resulted in more data from an improved diversity of languages.

For 52 of our languages, MS-All either matches or surpasses the number of Wikipedia pages for the language in question, showing the importance of the full dataset simply as raw data. In fact, the majority of MassiveSumm languages from South Saharan Africa (14/18) have more documents in MS-All than in Wikipedia. And well over half of the MassiveSumm languages for Eurasia (38/63) have more documents in MS-All than in Wikipedia.

Turning to Common Crawl, almost half of the languages from South Saharan Africa (8/18) have more pages in MS-All than in Common Crawl. Six out of 63 Eurasian languages have more articles in MS-All than in Common Crawl.

When we consider even just the heavily filtered automatic summarisation portion of the data, MS, we find that 10 of the South Saharan African languages contain more pages than Wikipedia, and 5 out of 18 of these languages contain more data than Common Crawl. For Eurasia, 19 of the 63 languages contain more pages than Wikipedia.

Table 5 gives the proportions of the articles in MS-All that are also contained in Common Crawl, for those languages where more than $49 \%$ can be obtained. This is 18 languages-around a fifth of the languages represented by MassiveSumm. Hence observe that large portions of easily indexible and crawlable, publicly available, diverse linguistic data are not being scraped into one of the most important datasets for NLP, both in size, but in determining to a large extent which languages get mainstream NLP research: Common Crawl.

\section{Reflections on Low-Resource Language Automatic Summarisation}

The central datasets for automatic summarisation have consistently been for English. In this section we consider how this focus on English has resulted in limited dataset curation methodology development (Section 5.1) and limited automatic summarisation system design (Section 5.2).

\footnotetext{
${ }^{5}$ April $2021 \quad$ crawl CC-MAIN-2021-04 https://commoncrawl.github.io/ cc-crawl-statistics/plots/languages.csv
} 


\begin{tabular}{|c|c|c|c|c|c|c|c|c|c|}
\hline family & | MS-All & $\%$ (MS-All) & MS & $\%(\mathbf{M S})$ & num langs & MS-All ave & MS-All ave \% & MS ave & MS ave\% \\
\hline Dravidian & 2005933 & $6.95 \%$ & 333765 & $2.7 \%$ & 4 & 501483.25 & $1.74 \%$ & 83441.25 & $0.67 \%$ \\
\hline Altaic & 835649 & $2.89 \%$ & 362191 & $2.93 \%$ & 5 & 167129.8 & $0.58 \%$ & 72438.2 & $0.59 \%$ \\
\hline Austro-Asiatic & 477331 & $1.65 \%$ & 257197 & $2.08 \%$ & 2 & 238665.5 & $0.83 \%$ & 128598.5 & $1.04 \%$ \\
\hline Niger-Congo & 467630 & $1.62 \%$ & 142921 & $1.16 \%$ & 10 & 46763.0 & $0.16 \%$ & 14292.1 & $0.12 \%$ \\
\hline Tai-Kadai & 252073 & $0.87 \%$ & 132287 & $1.07 \%$ & 2 & 126036.5 & $0.44 \%$ & 66143.5 & $0.53 \%$ \\
\hline Kartvelian & 182743 & $0.63 \%$ & 132055 & $1.07 \%$ & 1 & 182743.0 & $0.63 \%$ & 132055.0 & $1.07 \%$ \\
\hline Japanese & 125625 & $0.44 \%$ & 87220 & $0.71 \%$ & 1 & 125625.0 & $0.44 \%$ & 87220.0 & $0.71 \%$ \\
\hline other & 20120 & $0.07 \%$ & 8550 & $0.07 \%$ & 2 & 10060.0 & $0.03 \%$ & 4275.0 & $0.03 \%$ \\
\hline Mande & 1438 & $0.0 \%$ & 1 & $0.0 \%$ & 1 & 1438.0 & $0.0 \%$ & 1.0 & $0.0 \%$ \\
\hline Aymaran & 795 & $0.0 \%$ & 589 & $0.0 \%$ & 1 & 795.0 & $0.0 \%$ & 589.0 & $0.0 \%$ \\
\hline
\end{tabular}

Table 3: Language family-wise article counts and proportions for MassiveSumm-All (All) and for the MassiveSumm automatic summarisation dataset (MS).

\subsection{Impact on dataset curation}

The methodology we use for acquiring this dataset is based on Newsroom (Grusky et al., 2018), a dataset for English. In order for the method to be effective at obtaining data, at least the following two assumptions must be met.

Assumption 1. Digitalisation. Digitised newswire text must be publicly available online for the language, and in sufficiently large quantities. This is not the case, however. For example, a broad manual search for online news platforms in Africa $^{6}$ revealed relatively few non-colonial language platforms for the region. Digitised newswire is also sparse or non-existent in, for example, non-standard Arabic dialects, European languages such as Irish or Welsh, as well as indigenous languages in North and South America, and Australia. Hence focus on a strategy created for a language where there are massive amounts of online data, and lack of development of new techniques to acquire data for languages that do not have such an online presence will reinforce the lack of representation of these languages in automatic summarisation research.

Assumption 2. Web page structure conventions. Online news platforms must ensure that their article mark-ups abide by the Open Graph protocol (Cf. Section 3). However, extensive manual inspection revealed that while this is the norm for English and in general for languages of rich western countries, this is not the norm in general. For instance, due to this problem we had to exclude a number of other South Saharan African languages including

\footnotetext{
${ }^{6}$ https://www.w3newspapers.com/africa/
}

Southern Sotho, Pulaar, Zulu, and Luganda. Further, as we observe in Table 1, approximately 2 million documents are excluded from MS due to their summaries being empty-the news platforms in the corresponding languages have the correct template structure for their web pages, but do not use them as intended.

In order to develop the know-how to achieve true language diversity in datasets for automatic summarisation (and other NLP tasks), methods for acquiring automatic summarisation data should be developed which do not make these two assumptions. The difference in existence and in quantities of data for the languages of MassiveSumm reflect this requirement, which currently favours Indo-European languages.

\subsection{Systems: Low-resource baselines}

MassiveSumm provides a means to check whether there is evidence of some impact of a focus on English data for neural automatic summarisation.

The languages. We consider a minimal set of non-Indo-European languages to provide such evidence according to three separate considerations: (1) The languages should have large native speaker populations. . . (2) The languages should be nonIndo-European. (3) The set of languages should exhibit different complexity in morphology. (4) The datasets should be of significantly different sizes. (5) Finally, all languages must have readily available word segmentisers.

The set of languages we chose for our experiments all have a population far beyond that of the

\footnotetext{
${ }^{7}$ According to https://en.wikipedia.org/ wiki/List_of_languages_by_number_of_ native_speakers
} 


\begin{tabular}{|c|c|c|c|c|c|c|c|c|c|}
\hline language & family & MS & MS-all & Wiki & $\mathrm{CC}$ & MS/Wiki & MS/CC & MS-All/Wiki & MS-All/CC \\
\hline Amharic & Afro-Asiatic & 45,299 & 72,485 & 14,910 & 95,305 & $303.82 \%$ & $47.53 \%$ & $486.15 \%$ & $76.06 \%$ \\
\hline Fulah & Niger-Congo & 40 & 499 & 278 & 0 & $14.39 \%$ & - & $179.50 \%$ & - \\
\hline Hausa & Afro-Asiatic & 106,366 & 210,855 & 6,829 & 54,355 & $1557.56 \%$ & $195.69 \%$ & $3087.64 \%$ & $387.92 \%$ \\
\hline Igbo & Niger-Congo & 4,341 & 5,085 & 2,084 & 9,728 & $208.30 \%$ & $44.62 \%$ & $244.00 \%$ & $52.27 \%$ \\
\hline Oromo & Afro-Asiatic & 5,816 & 14,926 & 1,050 & 15,432 & $\mathbf{5 5 3 . 9 0 \%}$ & $37.69 \%$ & $1421.52 \%$ & $96.72 \%$ \\
\hline Rundi & Niger-Congo & 3,646 & 24,085 & 618 & 2,882 & $589.97 \%$ & $126.51 \%$ & $3897.25 \%$ & $835.70 \%$ \\
\hline Shona & Niger-Congo & 8,351 & 21,551 & 6,660 & 11,559 & $125.39 \%$ & $72.25 \%$ & $323.59 \%$ & $186.44 \%$ \\
\hline Somali & Afro-Asiatic & 26,738 & 186,605 & 5,944 & 159,270 & $449.83 \%$ & $16.79 \%$ & $3139.38 \%$ & $117.16 \%$ \\
\hline Swahili & Niger-Congo & 99,803 & 292,346 & 60,725 & 243,070 & $164.35 \%$ & $41.06 \%$ & $481.43 \%$ & $120.27 \%$ \\
\hline Tigrinya & Afro-Asiatic & 7,978 & 18,533 & 208 & 25,369 & $3835.58 \%$ & $31.45 \%$ & $8910.10 \%$ & $73.05 \%$ \\
\hline Xhosa & Niger-Congo & 172 & 12,876 & 1,182 & 37,430 & $14.55 \%$ & $0.46 \%$ & $1089.34 \%$ & $34.40 \%$ \\
\hline Armenian & Indo-European & 168,453 & 807,817 & 281,101 & $1,050,372$ & $59.93 \%$ & $16.04 \%$ & $287.38 \%$ & $76.91 \%$ \\
\hline Azerbaijani & Altaic & 140,685 & 301,134 & 177,955 & $1,548,046$ & $79.06 \%$ & $9.09 \%$ & $169.22 \%$ & $19.45 \%$ \\
\hline Bengali & Indo-European & 124,351 & 191,712 & 103,686 & $2,681,993$ & $119.93 \%$ & $4.64 \%$ & $184.90 \%$ & $7.15 \%$ \\
\hline Bosnian & Indo-European & 45,575 & 254,737 & 84,968 & $1,311,659$ & $53.64 \%$ & $3.47 \%$ & $299.80 \%$ & $19.42 \%$ \\
\hline Bulgarian & Indo-European & 667,560 & 955,497 & 269,103 & $9,070,911$ & $248.07 \%$ & $7.36 \%$ & $355.07 \%$ & $10.53 \%$ \\
\hline Central Khmer & Austro-Asiatic & 45,758 & 89,606 & 8,230 & 300,772 & $555.99 \%$ & $15.21 \%$ & $1088.77 \%$ & $29.79 \%$ \\
\hline Czech & Indo-European & 551,443 & 609,257 & 473,960 & $36,586,487$ & $116.35 \%$ & $1.51 \%$ & $128.55 \%$ & $1.67 \%$ \\
\hline Dari & Indo-European & 20,220 & 59,199 & 0 & 0 & - & - & - & - \\
\hline Georgian & Kartvelian & 132,055 & 182,743 & 148,069 & $1,269,380$ & $89.18 \%$ & $10.40 \%$ & $123.42 \%$ & $14.40 \%$ \\
\hline Gujarati & Indo-European & 43,830 & 450,740 & 29,481 & 294,393 & $148.67 \%$ & $14.89 \%$ & $1528.92 \%$ & $153.11 \%$ \\
\hline Hindi & Indo-European & 563,477 & $1,067,126$ & 145,723 & $4,185,074$ & $386.68 \%$ & $13.46 \%$ & $732.30 \%$ & $25.50 \%$ \\
\hline Hungarian & Uralic & 622,385 & 868,417 & 483,555 & $18,592,776$ & $128.71 \%$ & $3.35 \%$ & $179.59 \%$ & $4.67 \%$ \\
\hline Kannada & Dravidian & 47,676 & 281,630 & 26,789 & 309,943 & $177.97 \%$ & $15.38 \%$ & $1051.29 \%$ & $90.87 \%$ \\
\hline Oriya & Indo-European & 28,582 & 388,961 & 15,592 & 122,957 & $183.31 \%$ & $23.25 \%$ & $2494.62 \%$ & $316.34 \%$ \\
\hline Panjabi & Indo-European & 83,147 & 322,520 & 35,218 & 168,347 & $236.09 \%$ & $49.39 \%$ & $915.78 \%$ & $191.58 \%$ \\
\hline Persian & Indo-European & 529,924 & $1,134,376$ & 767,776 & $20,893,043$ & $69.02 \%$ & $2.54 \%$ & $147.75 \%$ & $5.43 \%$ \\
\hline Pushto & Indo-European & 58,038 & 215,927 & 11,807 & 90,702 & $491.56 \%$ & $63.99 \%$ & $1828.80 \%$ & $238.06 \%$ \\
\hline Scottish Gaelic & Indo-European & 15,012 & 16,528 & 15,198 & 48,315 & $98.78 \%$ & $31.07 \%$ & $108.75 \%$ & $34.21 \%$ \\
\hline Sinhala & Indo-European & 12,252 & 32,851 & 16,818 & 215,962 & $72.85 \%$ & $5.67 \%$ & $195.33 \%$ & $15.21 \%$ \\
\hline Slovak & Indo-European & 78,639 & 581,873 & 235,863 & $12,240,989$ & $33.34 \%$ & $0.64 \%$ & $246.70 \%$ & $4.75 \%$ \\
\hline Tamil & Dravidian & 45,239 & 885,408 & 134,646 & $1,444,153$ & $33.60 \%$ & $3.13 \%$ & $657.58 \%$ & $61.31 \%$ \\
\hline Telugu & Dravidian & 119,282 & 204,294 & 70,641 & 573,248 & $168.86 \%$ & $20.81 \%$ & $289.20 \%$ & $35.64 \%$ \\
\hline Thai & Tai-Kadai & 91,971 & 198,880 & 142,059 & $11,108,049$ & $64.74 \%$ & $0.83 \%$ & $140.00 \%$ & $1.79 \%$ \\
\hline Tibetan & Sino-Tibetan & 1,236 & 6,455 & 5,949 & 32,107 & $20.78 \%$ & $3.85 \%$ & $108.51 \%$ & $20.10 \%$ \\
\hline Ukrainian & Indo-European & 594,415 & $1,222,182$ & $1,073,297$ & $12,688,368$ & $55.38 \%$ & $4.68 \%$ & $113.87 \%$ & $9.63 \%$ \\
\hline Urdu & Indo-European & 621,738 & $1,096,319$ & 160,631 & 725,101 & $387.06 \%$ & $85.75 \%$ & $682.51 \%$ & $151.20 \%$ \\
\hline Welsh & Indo-European & 53,802 & 154,844 & 132,464 & 358,792 & $40.62 \%$ & $15.00 \%$ & $116.90 \%$ & $43.16 \%$ \\
\hline
\end{tabular}

Table 4: Languages for which MassiveSumm carries more raw documents than Wikipedia or Common Crawl.

average European country. And yet two of these languages are severely lower resourced in NLP in general, if not zero-resourced. The languages are:

- Arabic, a semitic language with a complex morphology and around 310 million native speakers. We used 432,384 article-summary pairs from MS.

- Telugu, a Dravidian language with a moderately rich morphology and around 82 million native speakers. We used 12,633 articlesummary pairs from MS.

- Hausa, an Afro-Asiatic tonal language with a relatively simple morphology and around
43 million native speakers. We used 78,633 article-summary pairs from MS.

The datasets were split into train/test/dev sets with corresponding proportions $80 \% / 10 \% / 10 \%$. For tokenisation of Arabic and Telugu we used Spacy (Honnibal et al., 2020), and the English tokeniser from NLTK (Loper and Bird, 2002) for Hausa. For sentence segmentation we use PySBD (Sadvilkar and Neumann, 2020) for Arabic, and NLTK for the remaining Hausa and Telugu.

The system. OpenNMT's (Klein et al., 2017) reimplementation of the Pointer-Generator system (See et al., 2017) provides efficient state-of-the- 


\begin{tabular}{lc|lc}
\hline language & $\boldsymbol{\%}$ & language & $\boldsymbol{\%}$ \\
\hline Tibetan & $96.98 \%$ & Lingala & $72.05 \%$ \\
Lao & $95.45 \%$ & Malagasy & $67.18 \%$ \\
Bambara & $95.37 \%$ & Tigrinya & $66.69 \%$ \\
Dari & $94.87 \%$ & Bosnian & $63.71 \%$ \\
Rundi & $84.28 \%$ & Scot. Gaelic & $63.71 \%$ \\
Burmese & $81.51 \%$ & Hungarian & $61.30 \%$ \\
Haitian & $79.50 \%$ & Slovenian & $58.21 \%$ \\
Oromo & $77.93 \%$ & Bislama & $50.00 \%$ \\
Kurdish & $74.77 \%$ & Irish & $49.27 \%$ \\
\hline
\end{tabular}

Table 5: Languages from MassiveSumm-All for which the percentage of articles that can also be found in Common Crawl is greater than $49 \%$.

art-competitive performance and proved more robust to limits in dataset size than a Transformer (Vaswani et al., 2017) model during our hyperparameter search preparatory experiments-this was a crucial requirement for our low-resource language experiments. We experimented with training both Pointer-Generator and Transformer models over different quantities, $20 \%$ and $100 \%$ (respectively, 57,444 and 287,227 instances), of CNNDM training data. While the transformer outperforms PG when training on the full dataset (Table 6), it grossly overfits when faced with only $20 \%$ of the data for training (Figure 1).

\begin{tabular}{l|ccc}
\hline \multicolumn{1}{c|}{ system (train prop.) } & R1 & R2 & RL \\
\hline Transformer (100\% data) & 39.06 & 17.02 & 36.09 \\
Transformer (20\% data) & 32.23 & 11.12 & 29.99 \\
\hline PG (100\%) & 38.41 & 16.31 & 35.21 \\
PG (80\%) & 38.18 & 16.3 & 35.08 \\
PG (60\%) & 38.13 & 16.16 & 34.92 \\
PG (40\%) & 38.05 & 16.13 & 34.9 \\
PG (20\%) & 36.81 & 15.36 & 33.7 \\
\hline
\end{tabular}

Table 6: Rouge-1, Rouge-2, and Rouge-L (Lin, 2004) scores for comparing Transformer and RNN (PG) models on different proportions of CNNDM training data in preparation for lower-resource language experiments.

For further context, we also train and test on the Newsroom corpus. Since the Newsroom corpus did not filter prefix and ellipsis summaries, we include scores with and without these data filters. We use an $80 \% / 10 \% / 10 \%$ split of Newsroom before and after filtering: respectively 994,446/109,147/109,147 and $808,727 / 88,657 / 88,768$ article-summary pairs.

During training we truncate articles to 400 tokens and summaries to 100 tokens. We fix the

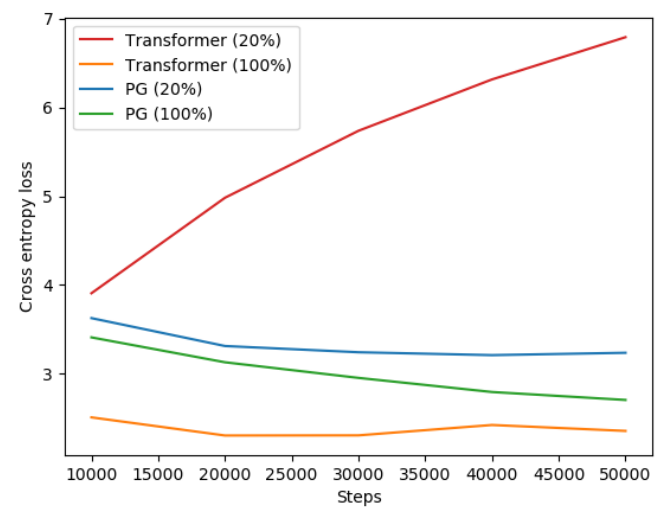

Figure 1: Two fixed architecture configurations run under two data settings: (1) $100 \%$ of the training set, and (2) $20 \%$ of the training set. The PG model (rnn) is robust to different data settings while the transformer quickly overfits the training data. Loss in the graph is measured over the development set.

random seed but refrain from tying the input and output embeddings (Press and Wolf, 2016). The vocabularies are fixed to 30,000 tokens across all languages and we used no subword tokeniser. At inference time we decoded with a beam size of 10, discarded summaries with less than 35 tokens, block trigrams and apply length penalty with the value $\alpha=0.9$ (Wu et al., 2016). For further details of the model, we refer to the original papers of (See et al., 2017; Gehrmann et al., 2018) as well as OpenNMT's documentation ${ }^{8}$. Our experiments should act as lower bounds as we conducted no tuning on any of the MassiveSumm datasets.

We include the Lead-3 baseline which simply copies the first three sentences from the article. It is a notoriously strong baseline for automatic summarisation systems and acts as a baseline point of reference that is resilient to training set size limitations.

The results are given in Table 7. In particular, we notice that ROUGE scores tend to be rather low for the largest non-English dataset, Arabic, with the most complex morphology, despite being the largest of the three. As expected, Telugu with the smallest dataset, also has low ROUGE scores. On the other hand, Lead-3 performs better but similarly low in ROUGE score. On the other hand, ROUGE scores for Hausa are significantly higher in scale than Newsroom scores and also significantly outperform the strong Lead-3 baseline. We have 3

\footnotetext{
${ }^{8}$ https://opennmt. net/OpenNMT-py/ examples/Summarization.html
} 
different linguistic contexts and three quite different behaviours, which provides clear evidence that robust development in automatic summarisation must adjust and consider linguistic diversity.

\begin{tabular}{l|ccc}
\hline \multicolumn{1}{c|}{ dataset (system) } & R1 & R2 & RL \\
\hline Arabic (Point.-Gen.) & 13.58 & 4.02 & 13.53 \\
Arabic (Lead-3) & 11.34 & 3.18 & 11.27 \\
Hausa (Point.-Gen.) & 38.55 & 28.5 & 31.91 \\
Hausa (Lead-3) & 30.55 & 17.95 & 26.68 \\
Telugu (Point.-Gen.) & 5.62 & 1.43 & 5.62 \\
Telugu (Lead-3) & 8.87 & 2.17 & 8.7 \\
\hline Newsroom (Point.-Gen.) & 34.73 & 21.25 & 30.39 \\
Newsroom (Lead-3) & 31.12 & 21.4 & 28.49 \\
Filt. Newsroom (Point.-Gen.) & 28.95 & 15.5 & 23.9 \\
Filt. Newsroom (Lead-3) & 25.49 & 14.17 & 22.49 \\
\hline
\end{tabular}

Table 7: Baseline ROUGE scores for Arabic, Hausa, and Telugu. ROUGE scores for Newsroom added for context.

\section{Concluding Remarks}

In this paper, we presented the most large-scale, most language and linguistically diverse and inclusive dataset for automatic summarisation to date: MassiveSumm. In acquiring MassiveSumm, we also acquired one of the most diverse and inclusive sources of raw linguistic data to date. We also provided evidence how focus on anglo-centric data acquisition method development and system development were detrimental to both language inclusion and language agnostic system behaviour.

\section{References}

Jianpeng Cheng and Mirella Lapata. 2016. Neural summarization by extracting sentences and words. In Proceedings of the 54th Annual Meeting of the Association for Computational Linguistics (Volume 1: Long Papers), pages 484-494, Berlin, Germany. Association for Computational Linguistics.

Sebastian Gehrmann, Yuntian Deng, and Alexander Rush. 2018. Bottom-up abstractive summarization. In Proceedings of the 2018 Conference on Empirical Methods in Natural Language Processing, pages 4098-4109, Brussels, Belgium. Association for Computational Linguistics.

Google. 2018. Google patents public datasets: connecting public, paid, and private patent data.

Max Grusky, Mor Naaman, and Yoav Artzi. 2018. Newsroom: A dataset of 1.3 million summaries with diverse extractive strategies. In Proceedings of the 2018 Conference of the North American Chapter of the Association for Computational Linguistics: $\mathrm{Hu}$ man Language Technologies, Volume 1 (Long Papers), pages 708-719, New Orleans, Louisiana. Association for Computational Linguistics.

Tahmid Hasan, Abhik Bhattacharjee, Md. Saiful Islam, Kazi Mubasshir, Yuan-Fang Li, Yong-Bin Kang, M. Sohel Rahman, and Rifat Shahriyar. 2021. XLsum: Large-scale multilingual abstractive summarization for 44 languages. In Findings of the Association for Computational Linguistics: ACL-IJCNLP 2021, pages 4693-4703, Online. Association for Computational Linguistics.

Karl Moritz Hermann, Tomas Kocisky, Edward Grefenstette, Lasse Espeholt, Will Kay, Mustafa Suleyman, and Phil Blunsom. 2015. Teaching machines to read and comprehend. In Advances in Neural Information Processing Systems 28, pages 1693-1701. Curran Associates, Inc.

Matthew Honnibal, Ines Montani, Sofie Van Landeghem, and Adriane Boyd. 2020. spaCy: Industrial-strength Natural Language Processing in Python.

Baotian Hu, Qingcai Chen, and Fangze Zhu. 2015. LCSTS: A large scale Chinese short text summarization dataset. In Proceedings of the 2015 Conference on Empirical Methods in Natural Language Processing, pages 1967-1972, Lisbon, Portugal. Association for Computational Linguistics.

Guillaume Klein, Yoon Kim, Yuntian Deng, Jean Senellart, and Alexander Rush. 2017. OpenNMT: Opensource toolkit for neural machine translation. In Proceedings of ACL 2017, System Demonstrations, pages 67-72, Vancouver, Canada. Association for Computational Linguistics.

Chin-Yew Lin. 2004. ROUGE: A package for automatic evaluation of summaries. In Text Summarization Branches Out, pages 74-81, Barcelona, Spain. Association for Computational Linguistics.

Edward Loper and Steven Bird. 2002. Nltk: The natural language toolkit. In In Proceedings of the ACL Workshop on Effective Tools and Methodologies for Teaching Natural Language Processing and Computational Linguistics. Philadelphia: Association for Computational Linguistics.

Ramesh Nallapati, Bowen Zhou, Cicero dos Santos, Çağlar Gulçehre, and Bing Xiang. 2016. Abstractive text summarization using sequence-to-sequence RNNs and beyond. In Proceedings of The 20th SIGNLL Conference on Computational Natural Language Learning, pages 280-290, Berlin, Germany. Association for Computational Linguistics.

Shashi Narayan, Shay B. Cohen, and Mirella Lapata. 2018. Don't give me the details, just the summary! topic-aware convolutional neural networks for extreme summarization. In Proceedings of the 2018 
Conference on Empirical Methods in Natural Language Processing, pages 1797-1807, Brussels, Belgium. Association for Computational Linguistics.

Khanh Nguyen and Hal Daumé III. 2019. Global Voices: Crossing borders in automatic news summarization. In Proceedings of the 2nd Workshop on New Frontiers in Summarization, pages 90-97, Hong Kong, China. Association for Computational Linguistics.

Ofir Press and Lior Wolf. 2016. Using the output embedding to improve language models. arXiv preprint arXiv:1608.05859.

Nipun Sadvilkar and Mark Neumann. 2020. PySBD: Pragmatic sentence boundary disambiguation. In Proceedings of Second Workshop for NLP Open Source Software (NLP-OSS), pages 110-114, Online. Association for Computational Linguistics.

Evan Sandhaus. 2008. The new york times annotated corpus. Technical report, Linguistic Data Consortium, Philadelphia.

Thomas Scialom, Paul-Alexis Dray, Sylvain Lamprier, Benjamin Piwowarski, and Jacopo Staiano. 2020. MLSUM: The multilingual summarization corpus. In Proceedings of the 2020 Conference on Empirical Methods in Natural Language Processing (EMNLP), pages 8051-8067, Online. Association for Computational Linguistics.

Abigail See, Peter J. Liu, and Christopher D. Manning. 2017. Get to the point: Summarization with pointergenerator networks. In Proceedings of the 55th Annual Meeting of the Association for Computational Linguistics (Volume 1: Long Papers), pages 10731083, Vancouver, Canada. Association for Computational Linguistics.

Eva Sharma, Chen Li, and Lu Wang. 2019. BIGPATENT: A large-scale dataset for abstractive and coherent summarization. In Proceedings of the 57th Annual Meeting of the Association for Computational Linguistics, pages 2204-2213, Florence, Italy. Association for Computational Linguistics.

Daniel Varab and Natalie Schluter. 2020. DaNewsroom: A large-scale Danish summarisation dataset. In Proceedings of the 12th Language Resources and Evaluation Conference, pages 6731-6739, Marseille, France. European Language Resources Association.

Ashish Vaswani, Noam Shazeer, Niki Parmar, Jakob Uszkoreit, Llion Jones, Aidan N Gomez, Ł ukasz Kaiser, and Illia Polosukhin. 2017. Attention is all you need. In Advances in Neural Information Processing Systems, volume 30. Curran Associates, Inc.

Yonghui Wu, Mike Schuster, Zhifeng Chen, Quoc V Le, Mohammad Norouzi, Wolfgang Macherey, Maxim Krikun, Yuan Cao, Qin Gao, Klaus Macherey, et al. 2016. Google's neural machine translation system: Bridging the gap between human and machine translation. arXiv preprint arXiv:1609.08144.

\section{A Appendix}

This appendix contains the full version of Table 1. 


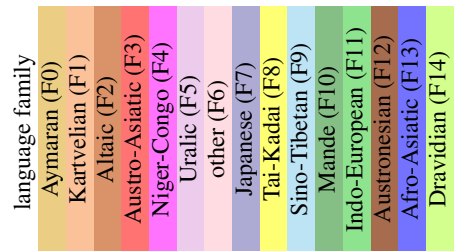

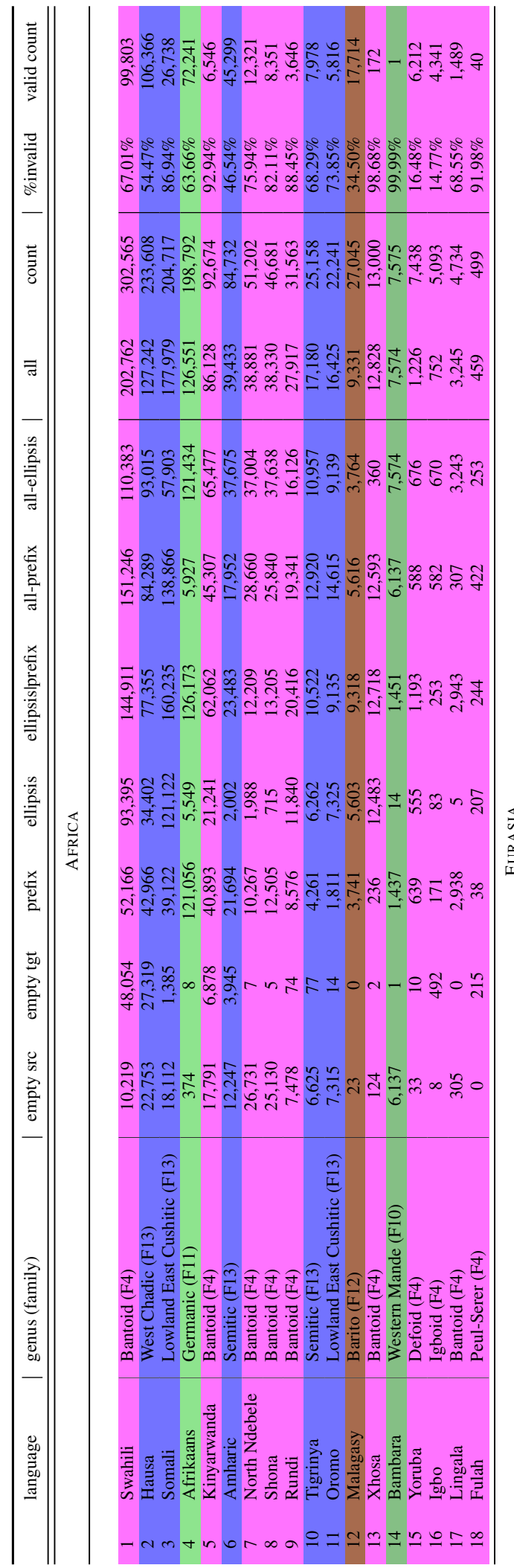

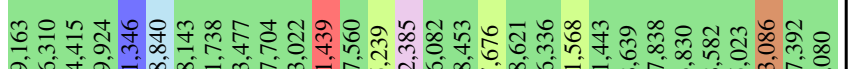

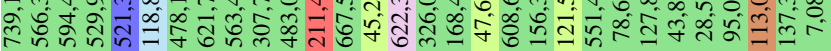

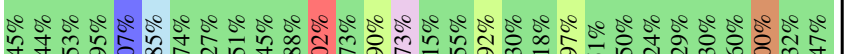

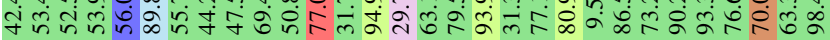

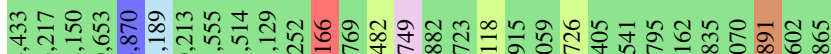

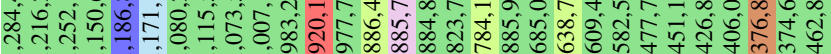

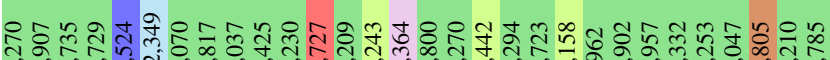

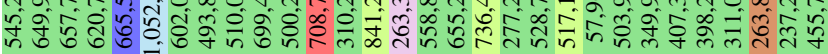

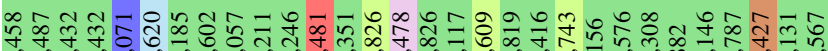
等

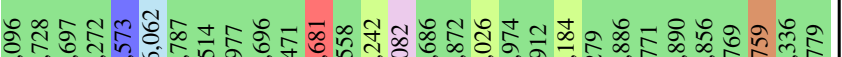

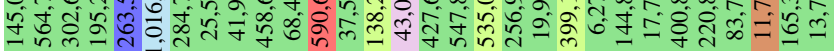

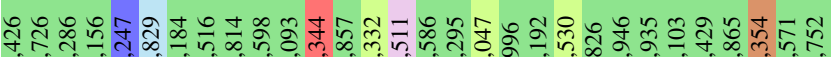

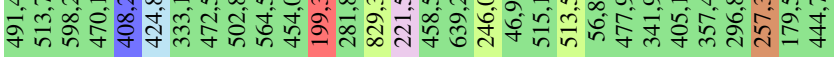

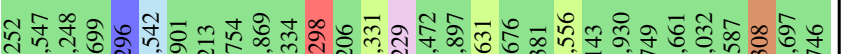

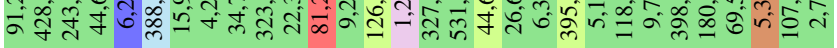

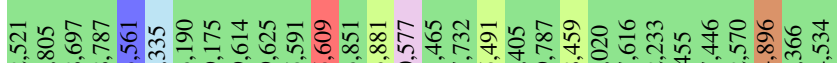

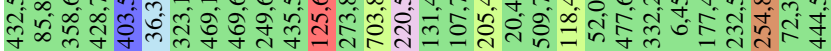

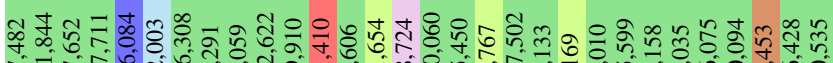

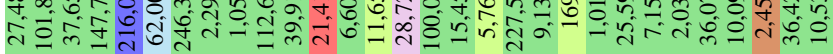

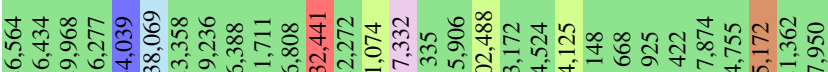

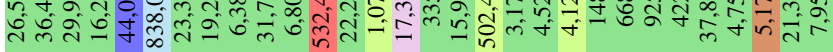

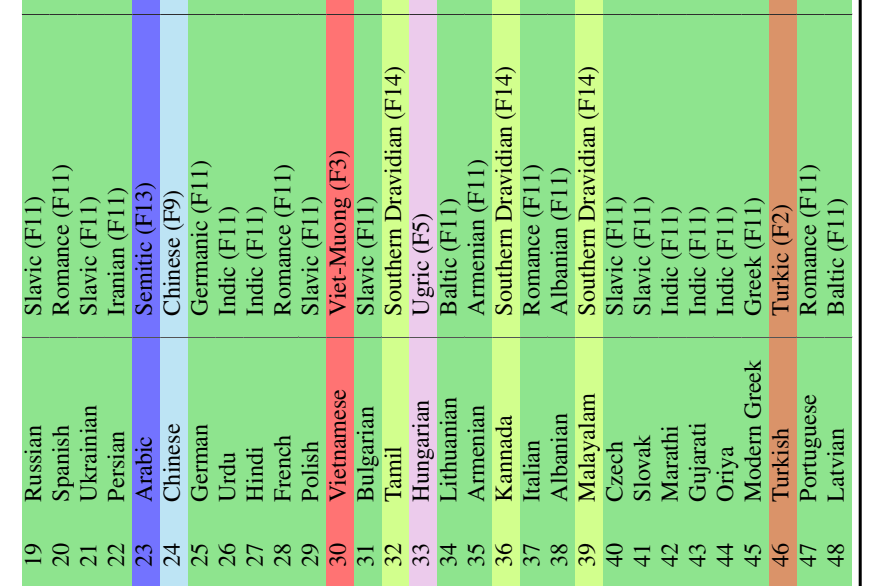

苛

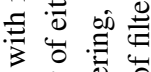
幽究

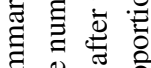

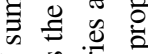

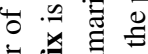

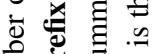

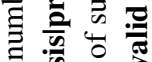
产若 을 娄

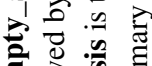
言产产 这递 要过

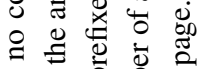

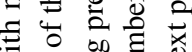

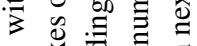
ช

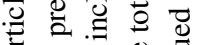

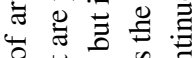
可施

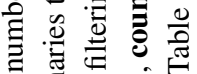
g $\therefore$ क

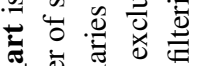
尼

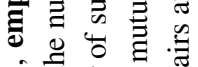
s.

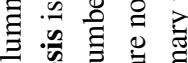

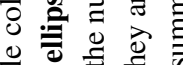
o d

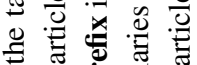

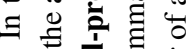

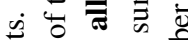

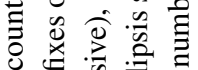
记家言

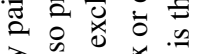

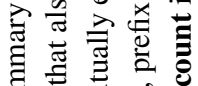

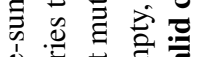
웡 氙 品 吅 它氞

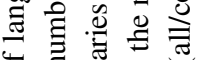
녕

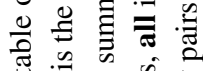
$\Xi$ 的

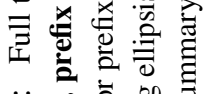
$\ddot{0}$ s to

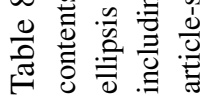



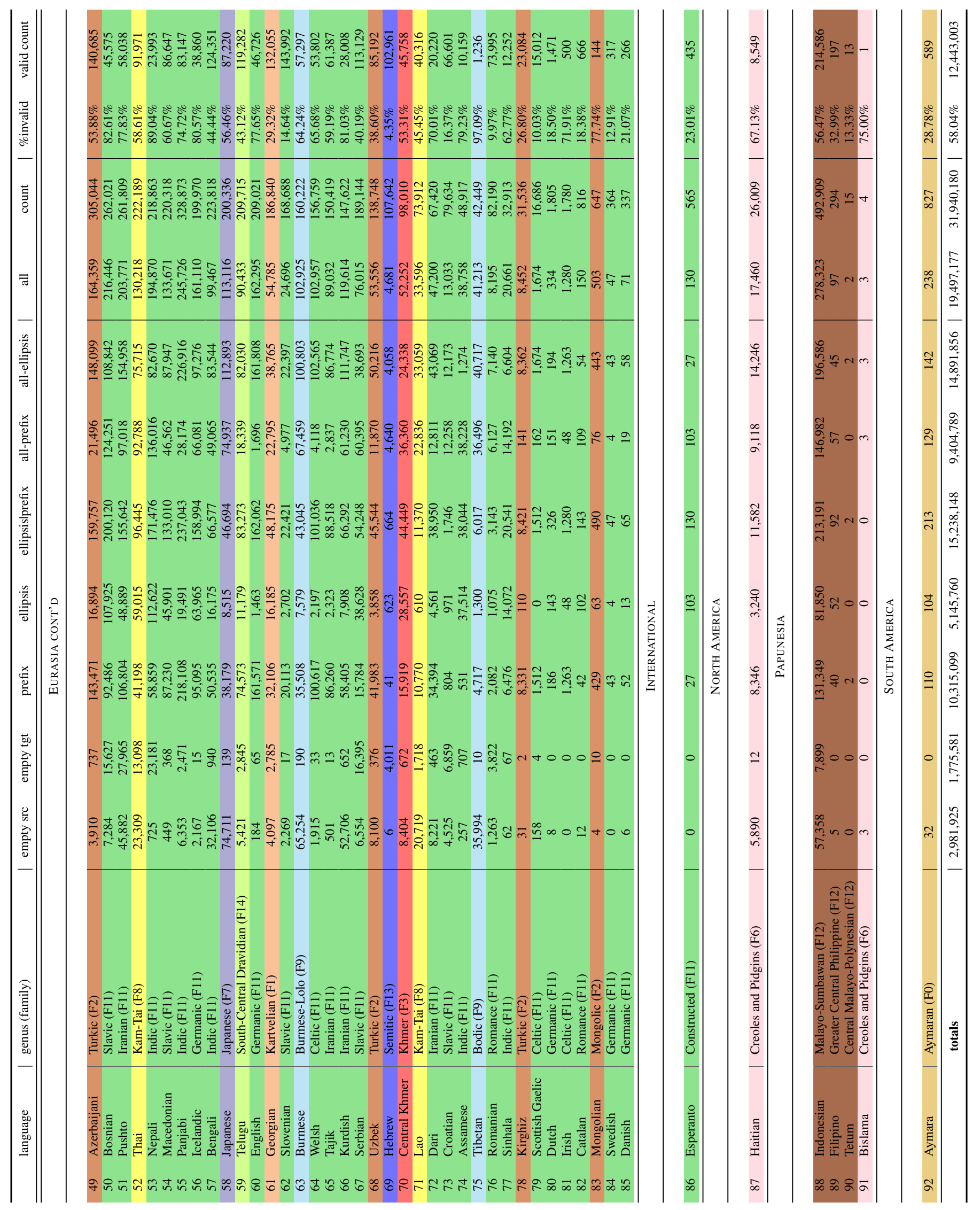